DEPARTAMENTO DE EMBRIOLOGIA E HISTOLOGIA DA FACULDADE DE MEDICINA DA UNIVERSIDADE DE SAO PAULO (DIRETOR: PROF. CARMO LORDY) E CADEIRA DE PEDIATRIA DA ESCOLA PAULISTA DE MEDICINA (DIRETOR: PROF. PEDRO DE ALCANTARA)

\title{
ANEMIA POR LEITE DE CABRA
}

\section{EMILIO MATTAR}

Assistente voluntario de Embriologia e Histologia. Medico interno da $2 .^{2}$ Medicina de Homens da Santa Casa.

\section{EWALDO MARIO RUSSO}

Assistente voluntario de Pediatria. Diretor clinico da Creche Santa Luzia.

O conceito da anemia por leite de cabra, em sua patogenia, alterações fundamentais hematopoieticas e hematologicas, não foi ainda claramente estabelecido. As anemias verificadas por alimentação com leite de cabra são, em geral, mais intensas do que a generalidade das chamadas anemias alimentares infantis. Dois grupos diferem extremamente em sua concepção quanto á natureza intima dos processos que originam esta hemopatia. A escola austro-alemã com Czerny, Glanzmann, Opitz, Kleinschmidt, interpreta o processo como resultante de toxicos alimentares existentes no leite de cabra. Estes autores, e suas respetivas escolas, focalizam como centro gerador das anemias alimentares, processos de natureza toxica. As determinantes causais estariam quer na maior riqueza em gorduras do leite de cabra, quer em acido graxos inferiores (acidos caprilicos), que seriam toxi-hemolizantes para o eritronio e trofo-toxícos para os parenquimas hematopoieticos, gerando, em ultima analise, hemopatias toxicas e hemoliticas, por excesso ou presença de gorduras inadequadas. No entanto, os fatores toxicos lateos não foram demonstrados, a hemolize toxica não ficou provada (Bass e coll.), a ausencia de gorduras não é essencial para a remoção da anemia, e o extrato hepatico aliado ao ferro parece ser a melhor terapeutica.

Um segundo grupo (György e- Rouminger sobretudo) estudando as anemias por leite de cabra, sob o ponto de vista clinico e experimental, interpretam-n'a como de etiologia carencial. Bomskov e Rouminger alimentando ratos exclusivamente com leite de cabra, obtiveram anemia hipercromica resistente ao tratamento ferrico e respondendo bem ao extrato hepatico. As observações experimentais receberam confirmação clinica por György e Spöre. György define a anemia por leite de cabra como anemia perniciosiforme por carencia de fator estrinsico de Castle. De fato, o leite de cabra é estremamente pobre em fator exogeno, necessario á formação do principio anti-pernicioso, alem de possuir quota minima de ferro (Shohl). 
As observações experimentais de Rouminger e coll. não foram confirmadas por Beard e Boggess, por Kohler, Elvehjem e Hart, e por outros AA., que não verificaram anemia macrocitica nem valor globular maior do que a unidade e que obtiveram melhoria somente com o ferro, crendo porisso ser a anemia exclusivamente ferripriva sem carater perniciosiforme.

Até agora, entretanto, não existe acordo na litëratura quanto á natureza intima do processo: carencia de fatores hematogenicos, ou excesso de fatores hemo-toxicos. Entre a maioria dos AA. modernos que acredita na etiologia carencial, ha os que admitem ser a anemia exclusivamente ferripriva, e os que equiparam-n'a ás anemias macrociticas, perniciosiformes, portanto hepatoprivas, em sentido láto.

A mesma discordancia existe quanto ao quadro morfologico, anatomo-patologico e hematico, da hemopatia por leite de cabra: as descrições experimentais e clinicas mostram ora uma anemia microcitica hipocromica, ora anemia macrocitica hipercromica megaloblastica ou não, ás vezes, uma anemia de aspeto aplastico, e mesmo uma mielose eritro-leucemoide, forma v. Jaksch-Hayem.

Entre nós não se conhece descrição das alterações hematopoieticas determinadas por alimentação exclusiva com leite de cabra. De grande interesse pela sua raridade, pelas acentuadissimas alterações medulares e sanguineas estudadas poucos dias antes do exito letal; pela comprovação de um quadro combinado pernicioso-apłástico, é um caso que tivemos ocasião de observar.

O. F., 12 mêses de idade. Sexo feminino.

Nascida de. parto normal a termo, até os 6 mêses de idade alimentou-se exclusivamente ao seio, sem horario; no segundo semestre de vida a alimentação se restringiu com exclusividade ao leite de cabra, em diluição mais ou menos apreciavel, regimen dietetico que mantem até esta data. Aos 7 ou 8 mêses de idade, começou a perder peso, emagrecimento que se acentuou progressivamente até atingir a redução atual de 4.535 grs., gravissima para sua idade. Evacua 6 vezes ao dia, fézes aquosas, esverdeadas, pouco catarrais. Intensa distrofia. Atrofia das papilas linguaes. Ausencia de hepato-esplenomegalia. Leve micro-poliadenia periferica. Mucosas muito descoradas. Estado geral caquético. Foi tratada com transfusões sanguineas e alimentação adequađa, tendo falecido de infeção pneumonica intercorrente.

Exame hematologico: Hemacias: 2.360 .000 por mmc. Leucocitos: 8.800 por mmc. Hemoglobina 4,06 grs. $(28 \%)$. Valor globular: 0;58. Diametrc medio: 7,50 micra. Plaquetas: 80.000 por mmc.

Metamielocito: $0 \%$; bastonete: $1,50 \%$; segmentado: $61,50 \%$; eosinofilo: $3,50 \%$; linfocito tipico: $29,0 \%$; linfocito leucocitoide : $0,50 \%$; linfocito atipico: $1,0 \%$; linfoblasto: $0 \%$; monocito: $3,0 \%$.

Serie vermelha: Anisocitose e poiquilocitose muito intensas. Foi encontrado grande numero de hemacias de dimensões muito menores do que as normais (microcitos), esféricas e hipercromicas, com carater de esferocitos. Predominam porem as hemacias grandes, muitas nitidamente hipercromicas e outras hipocromicas. Em consideravel proporção dos globulos vermelhos foram vistas formações nitidamente patológicas: granulações azurófilas e aneis de Cabot. Presença de eritroblastos e megaloglastos em numero consideravel. $\mathrm{Na}$ serie megaloblastica encontraram-se elementos com nucleo irregular; com estrangulamentos mais ou menos profundos; foram vistas mitoses atipicas (com pequeno: numero de cromosomas) e tambem figuras de amitose. Observam-se frequentes 
cariorrexis patológicas, com formação de numerosos fragmentos nucleares: outras vezes notam-se fórmas poli ou ortocromaticas com 3 ou 4 massas nuclea. res arredondadas e picnóticas. Um megaloblasto policromatófilo apresentava um bastonete àzurófilo.

Serie branca: Acentuada anisocitóse neutrofila, com predominância de fórmas de tamanho maior do que o normal. Nucleos com caracteres degenerativos, citoplasma frequentemente vacuolizado. O numero de plaquetas evidentemente baixo, correspondente à megacariopenia central (ver mielograma) . O numero global de leucocitos é baixo para o normal nesta idade (11.675 por mmc. de acordo com a tabela de Mattar e col.) além de que na câmara de contagem são determinados todos os elementos nucleados, de maneira que nic numero global de leucocitos estão incluidos os eritroblastos e megaloblasto numerosos qụe se verificaram no sangue periférico. Há pois nitida leucopenia.

Mielograma:

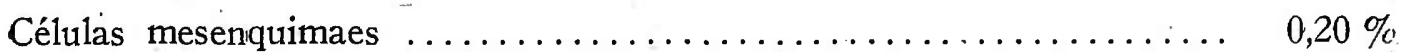

Hemocitoblastos $\ldots \ldots \ldots \ldots \ldots \ldots \ldots \ldots \ldots \ldots \ldots \ldots \ldots, 0,40 \%$

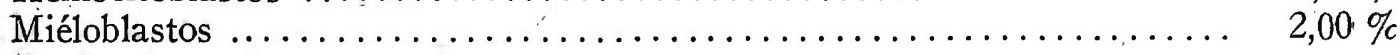

Neutrófilos

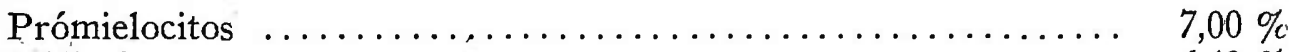

Miélocitos ....................................... $6,40 \%$

Metamielocitos ..................................... $9,00 \%$

Bastonetes ........................................ 2,60 \%

Segmentados $\ldots \ldots, \ldots \ldots \ldots \ldots \ldots \ldots \ldots \ldots \ldots \ldots \ldots \ldots \ldots \ldots \ldots, 3,20 \%$

Eosinófilos

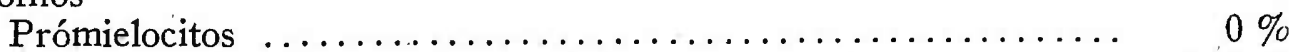

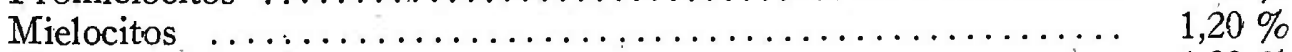

Metamielocitos $\ldots \ldots \ldots \ldots \ldots \ldots \ldots \ldots \ldots \ldots \ldots \ldots \ldots \ldots \ldots \ldots, 1,00 \%$

Bastonetes ......................................... $0,60 \%$

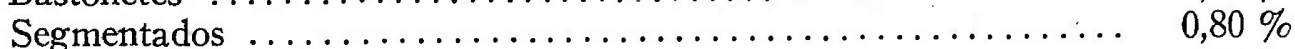

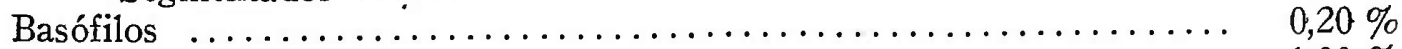

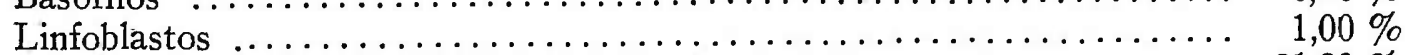

Linfocitos $\ldots \ldots \ldots \ldots \ldots \ldots \ldots \ldots \ldots \ldots \ldots \ldots \ldots \ldots \ldots \ldots \ldots \ldots \ldots \ldots \ldots, 21,80 \%$

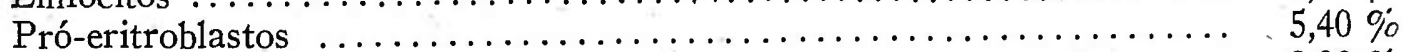

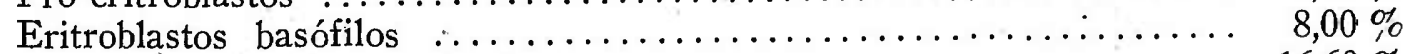

$" \quad$ policromaticos $\ldots \ldots \ldots \ldots \ldots \ldots \ldots \ldots \ldots \ldots \ldots, 16,60 \%$

$" \quad$ ortocromaticos $\ldots \ldots \ldots \ldots \ldots \ldots \ldots \ldots \ldots \ldots \ldots, 6,60 \%$

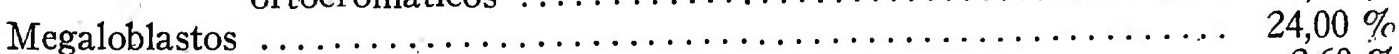

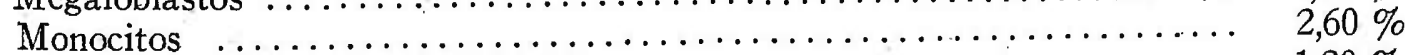

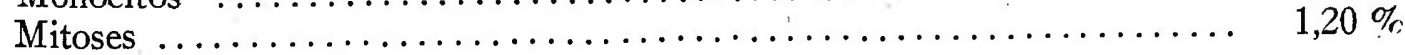

Medula fortemente hiperplastica na série vermelha, tanto normo como n:egaloblastica. Em ambas as linhagens, predominio nitido de fórmas basófilas e policromaticas, formando ninhos de proliferação, sem amadurecimento para células ortocromaticas. Atipias numerosas de cariorrexis, anisocitoses, etc., identicas às descritas no sangue periferico. Inibição da série mieloblastica, com sináis degenerativos em todas as fórmas evolutivas. Não foi encontrado megacarioblasto algum em todas as laminas examinadas.

São excepcionalmente raras, na hemopatia por leite de cabra, observações sobre alterações medulares estudadas pelo melhor metodo de verificação citologica que é o èsfregaço de material retirado por punção esternal. A hemopátia revela, como processo fundamental, comprometimento geral do tecido mieloide e se enquadra entre as mieloses applasticas globais na concepção de Di Guglielmo. A linhagem granulosa exibe aplasia e anaplasia; aplasia das celulas progenitoras, que se apresentam em numero diminuido; anaplasia destas 
mesmas celulas que perderam a capacidade de diferenciação citogenetica para celulas mais evolutivas, de tal maneira que o numero de granulosos maduros é muito baixo, mesmo menor que o numero de celulas granuloblasticas. Este achado - "Hemmung der Leucopoese" - verificado pela primeira vez por Letterer, é para este A. o fato mais constante da hemopatia por leite de cabra. A aplasia atinge tambem a serie megacarioblastica, correspondendo á plaquetopenia periferica. A serie vermelha alem da hiperplasia anaplastica (as celulas eritro e megaloblasticas constituem $60.6 \%$ de todas as celulas do esfregaço medular) exibe, como alteração essencial, notavel megaloblastose.

Na sistematização de Di Guglielmo, que tem a vantagem de definir a hemopatia pelas alterações do parenquima hematopoietico, este caso se enquadra melhor nas mieloses aplasticas globais, eritro-leucoplaquetopenicas com megaloblastose. Aliás este é o processo essencial na tipica anemia pernicosa, tipo Addison-Biermer; esta não pode ser definida pelo quadro clinico, pois a aquilia, a atrofia da mucosa lingual, as alterações de mielose funicular, etc. podem ser determinadas pelos tipos mais diversos de anemias primarias ou secundarias. O quadro periferico de anemia macrocitica não é exclusivo da anemia perniciosa, que aliás pode se apresentar como anemia macro e microcitica, hiper e hipocromica, As alterações medulares é que são especificas da anemia perniciosa, e deve-se notar que não só a megaloblastose define esta afecção, mas tambem uma tendencia aplastica de todas as celulas pertencentes á serie mieloide. A anemia perniciosa é, na verdade, uma mielose aplastica global, predominantemente eritroblastica, e com proliferação megaloblastica.

Uma megaloblastose central e periferica tão notavel como a exibida pelo caso referido só pode ser condicionada por falta de formação, absorção ou aproveitamento de principio antipernicioso. A anemia causada por alimentação exclusiva com leite de cabra não só é perniciosiforme, como diz György, mas pode ser classificada como anemia pernicosa, tipo Addison-Biermer, identica á observada no adulto. Por motivos intimos ignorados, a inibição da secreção do fator intrinsico de Castle, mecanismo mais comum na anemia perniciosa do adulto, é muito rara na criança. A carencia de fator extrinsico, no entanto, leva ás mesmas alterações hematopoieticas, desde que não se forma - assim como na insuficiente secreção de fator intrinsico o principio antipernicioso necessario á normal maturação dos eritroblastos.

Patogenicamente, pois, a anemia por leite de cabra deve ser interpretada como de natureza carencial, sendo a carencia tanto de principios nutritivos necessarios á inibição de megalogenese, como de fatores nutritivos necessarios á normal evolução do eritroblasto e á formação normal de hemoglobina (ferro sobretudo). Esta carencia mixta leva, em qualquer idade, á alterações hematicas proprias das anemias perniciosas hepatoprivas e das anemias hipocromicas fer- 


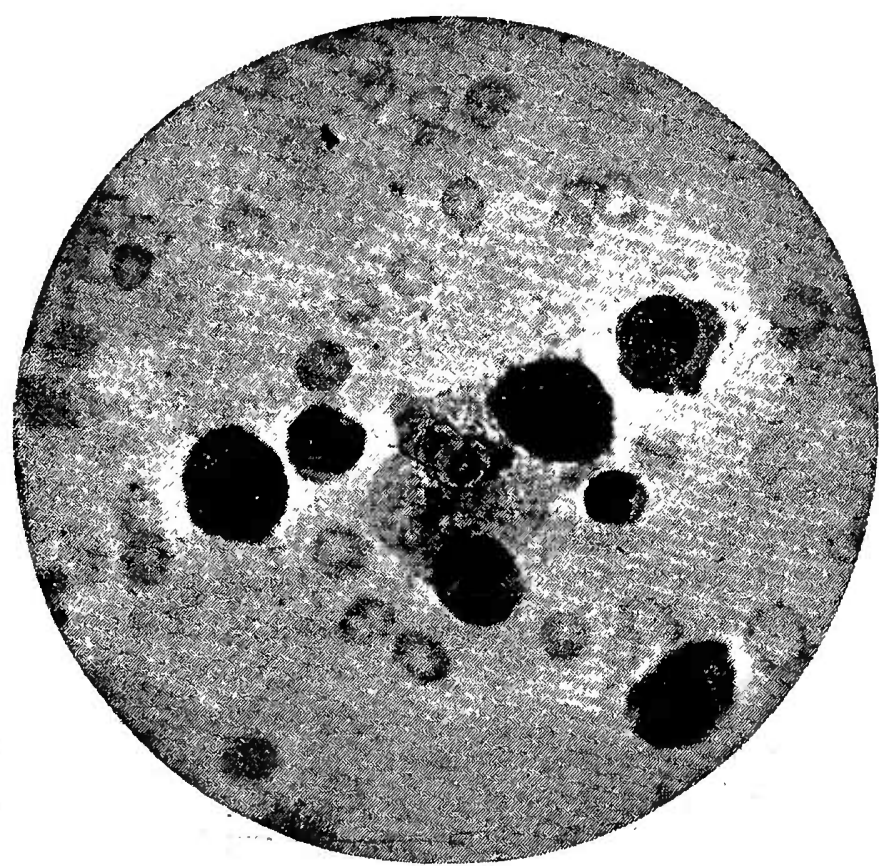

Fig. 1

Mielograma. Anemia por leite de cabra. Proliferaçāo megaloblastica.

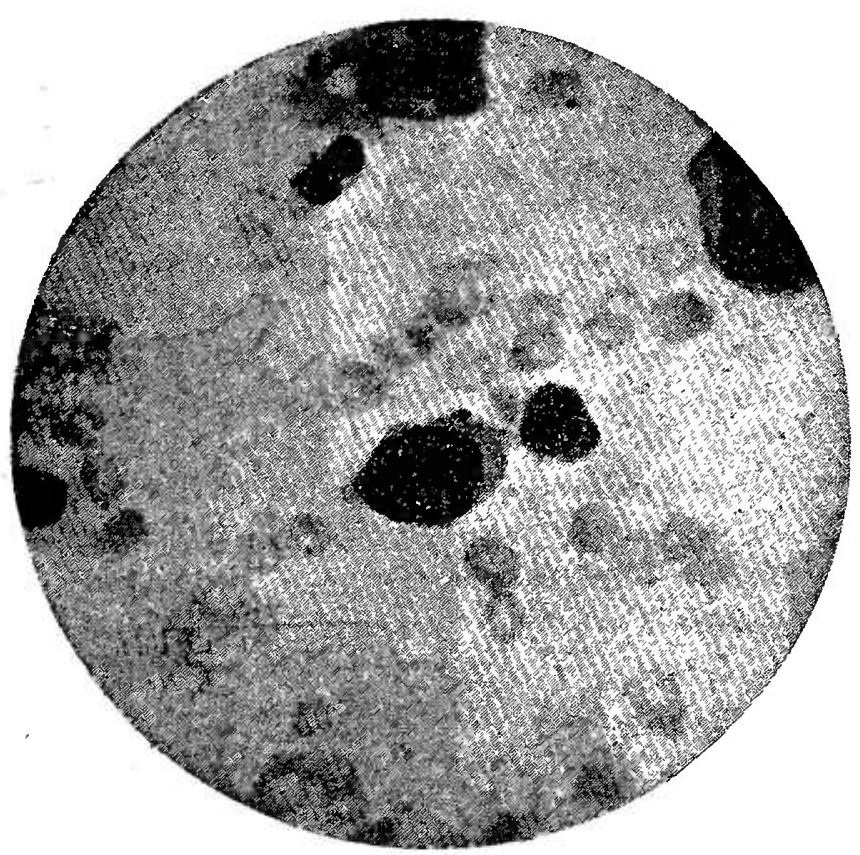

FIG. 2

Mielograma. Anemia por leite de cabra. Promegaloblasto. 


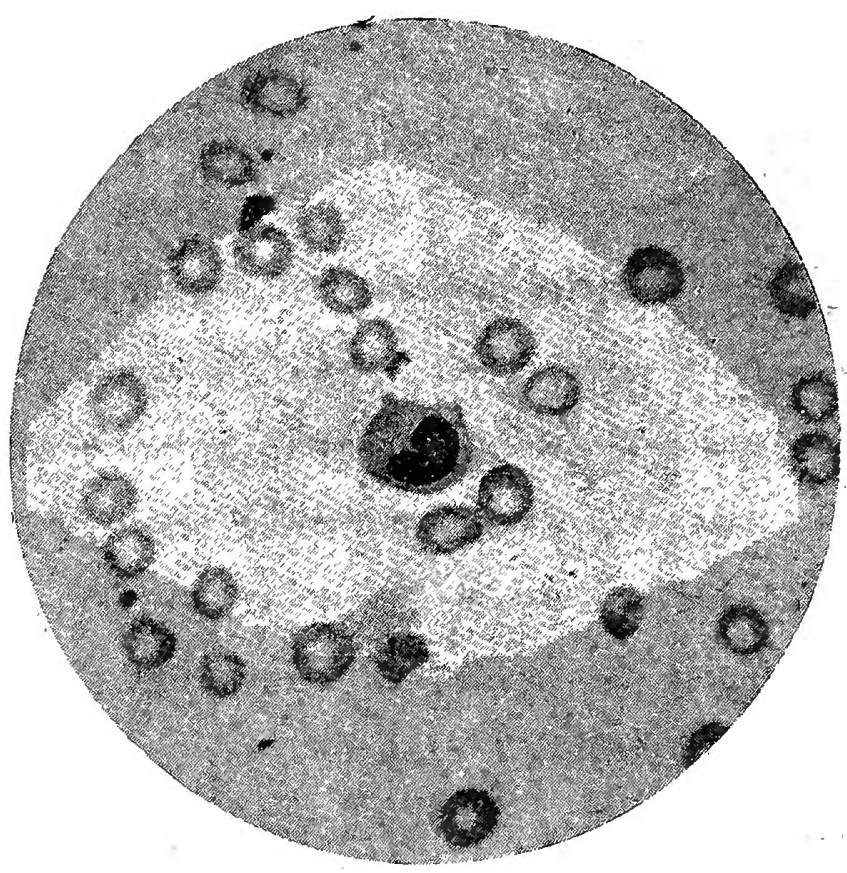

FIG. 3

Sangue periférico. Anemia por leite de cabra. Megaloblasto policromatófilo com bastonete azurófilo e nucleo lobado.

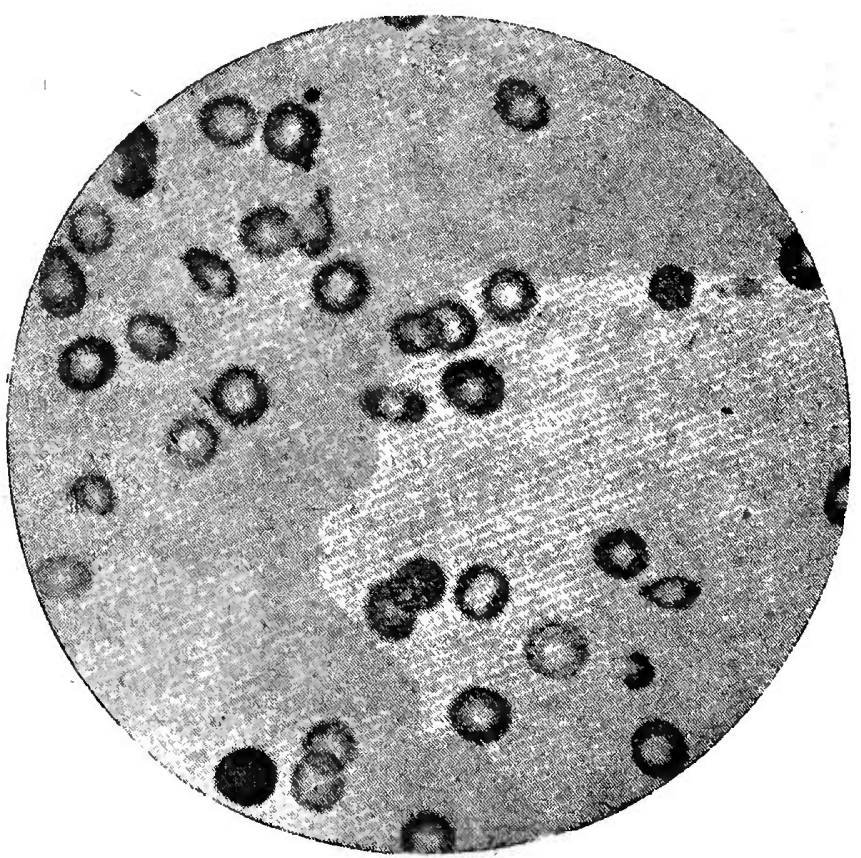

FIG. 4

Sangue periférico. Anemia por leite de cabra. Microcitia. esferocitia, policromasia, hipo e hipercromia. 


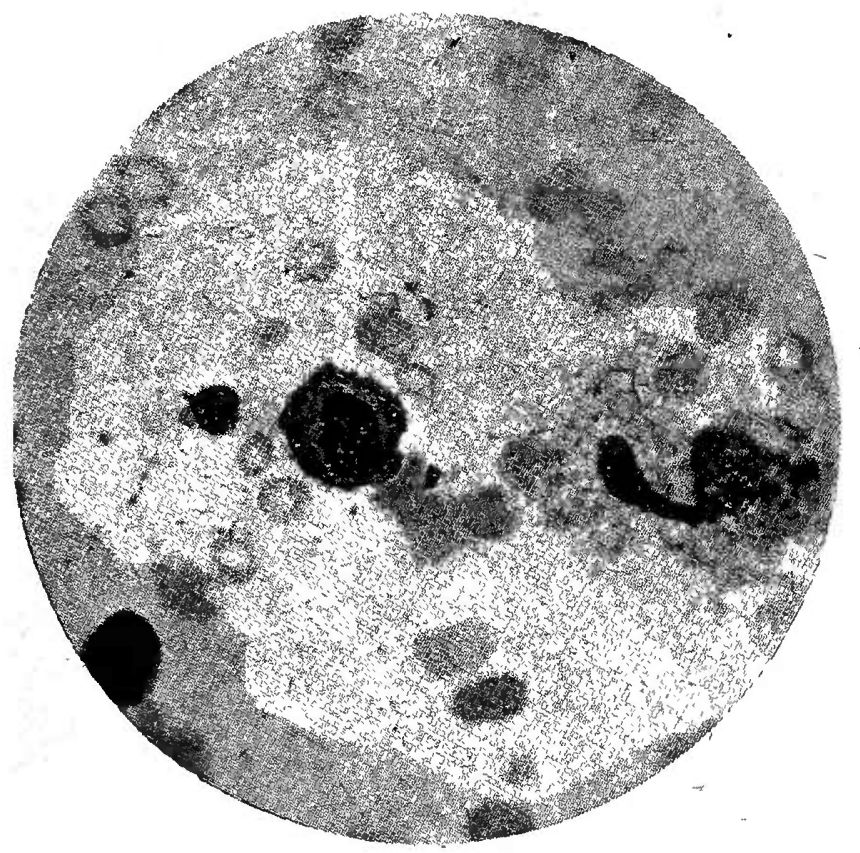

FIG. 5

Sangue periférico. Anemia por leite de cabra. Megaloblastu policromatico.

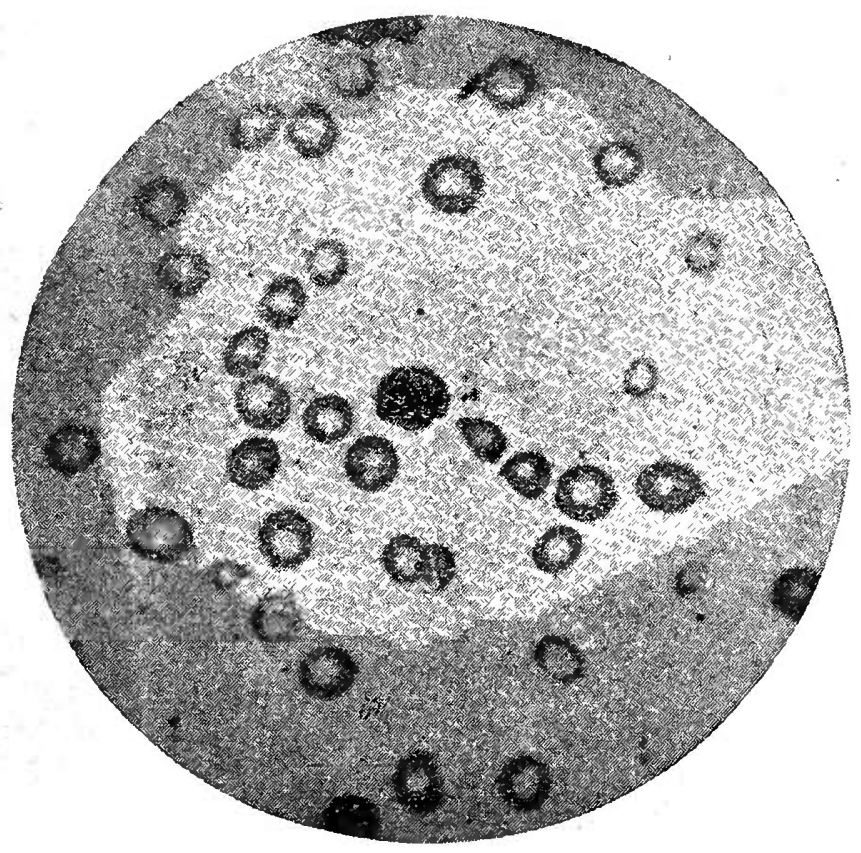

FIG. 6

Sangue periférico. Anemia por leite de cabra. Megaloblasto em cariorrexis patologica. Micro e macrocitos. 


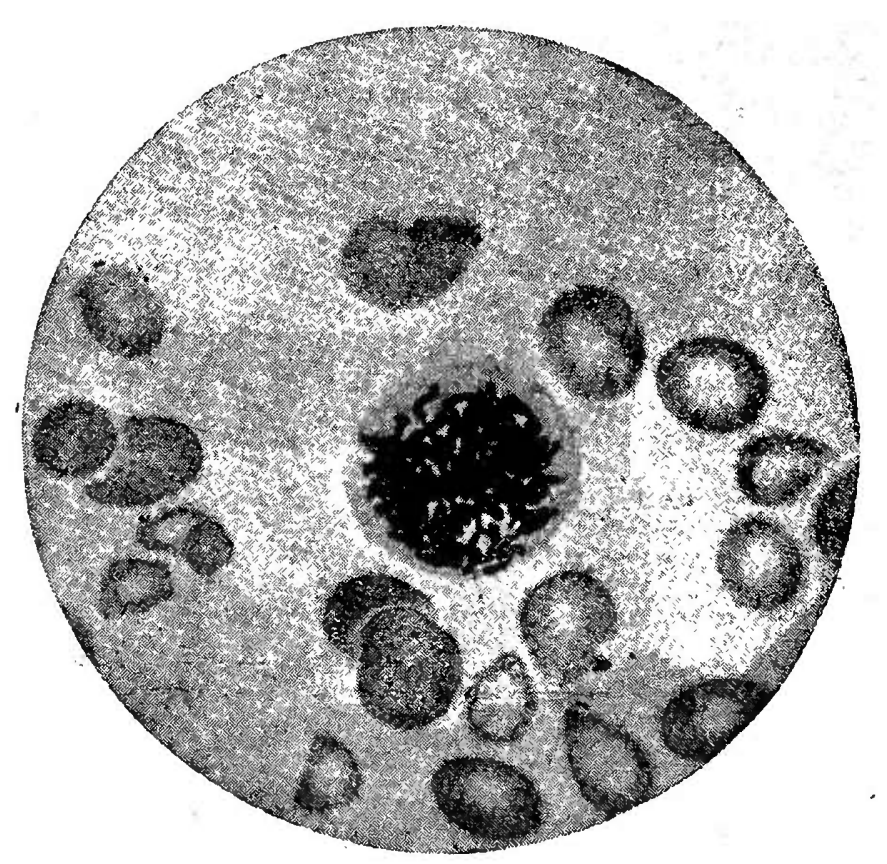

FIG. 7

Sangue periférico. Anemia por leite de cabra. Mitose atipica num grande megaloblasto ortocromatico. 
riprivas. São de nossa observação - como tambem de outros AA tipicas anemias perniciosas, tipo Addison-Biermer em que o valor globular está abaixo da unidade e o diametro medio é inferior ao normal devido á preponderante hipocromia e microcitia da adicional carencia ferrica. Porisso não falam contra a hipotese de anemia perniciosa o achado experimental ou clinico de hemaceás microciticas hipocromicas. Ao lado destas, expressão de carencia ferrica, encontram-se hemacias macrociticas hipercromicas, e, sobretudo a megaloblastose, alterações proprias da ausencia de principio anti pernicioso. A antiga teoria toxica de Naegeli para explicar as anemias perniciosa e perniciosiformes não encontra mais apoio nos fatos conhecidos atualmente, apesar da relutancia dos AA europeus em aceitar e adotar as novas concepções geradas das investigações da moderna escola americana de Whipple, Minot, Murphy, Castle. (Meulengracht). $O$ mesmo pode-se afirmar em relação às anemias alimentares infantis, inclusive a anemia por alimentação exclusiva com leite de cabra. Porisso, deve-se afastar a hipotese toxica defendida por, Opitz, Glanzmann, Czerny, etc.. Das alterações do metabolismo das gorduras - essencia patogenica da afecção para Glanzmann afigura-se-nos de importancia a diarreia gordurosa, que vai fazer aliar á insuficiente ingestão, uma deficiente absorção dos fatores nutritivos necessarios à hematopoiese.

Anatomicamente, o achado de proliferação megaloblastica ativissima, com todas as formas evolutivas do pro-megaloblasto ao megalocito, a hiperplasia sem amadurecimento das formas megalo e normoblasticas basofilas e policromaticas (anaplasia), a inibição granulopoietica e megacarioblastica, a leucopenia e a plaquetopenia periferica, a presença de sinais degenerativos na linhagem neutrofila, a macrocitose, a hipercromia, a aniso e a poiquilocitose, são comuns à hemopatia da alimentação por leite de cabra e à hemopatia perniciosa, tipo Addison-Biermer. A eritroblastose medular, a anaplasia eritoblastica, a hipocromia e a microcitia, exprimem a acentuada carencia ferrica concumitante desde que o leite de cabra tem quantidade de ferro 3 a 4 vezes menor que o leite humano (Shohl) já por si pobre em ferro. As alterações citologicas de esfregaços medulares demonstram que nestes casos, em que ha maxima carencia exogena, no sentido hematogenico, a mielose é acentuadamente aplastica.

Isto vem confirmar, em parte, as observações anatomo-patologicas de Letterer, em varios casos de anemia por leite de cabra, estudados sob o ponto de vista de sua morfologia patologica. A acentuada aplasia mieloide sugere que nestas anemias nutritivas falte - um fator dietetico, não conhecido ainda, necessario à leuco e megacariogenese; tal fator tem sido pesquizado e experimentado na terapeutica das mieloses aplasticas, tendo-se sugerido sua- presença; por Marburg, na medula gordurosa dos ossos. O quadro morfologico nem sempre corresponde ao da mielose perniciosa aplastica global; esta representa o gráo maximo de carencia, como no caso descrito, 
em que as alterações hematopoieticas foram estudadas 3 dias antes do exito letal. Si a alimentação exclusiva por leite de cabra é mantida por certo tempo, desenvolve-se inicialmente uma mielose eritropenica ferripriva, portanto hipocromica e microcitica. Temos observado mais de um caso neste estadio em que a terapeutica ferrica, por si só, faz regredir a anemia. Em geral, as alterações são verificaveis quando, ao lado da microcitia hipocromica, já existem macrocitos hipercromaticos. Neste periodo começa a se notar anaplasia na serie granulosa e megacarioblastica. Nos estadios mais avançados de carencia, a megaloblastose e a aplasia global predominam no processo. Morfologicamente, a intensificação progressiva da carencia nutritiva corresponde à passagem, tambem progressiva, da mielose parcial eritropenica para global leuco-megacario e eritropenica, predominantemente eritropenica. Não é, pois, de extranhar que, na literatura, tanto experimental como clinicamente, o quadro morfologico varie desde anemias simples, macro où microciticas, até mieloses globais aplasticas, megaloblasticas ou não. Esta aplasia global é a base anatomica do quadro clinico com purpuras cutaneas, hemorragias e ulcerações gengivais, atrofia da mucosa lingual, menor resistencia às infecções, quadro proprio de mielose aplastica.

\section{LITERATURA}

1 - BEARD H. H. e Boggess T. S. - A comparison of the anemia produced by feeding young rats upon human, cow and goat milk. Am. Jour. Physiol. 113, 642, 1935.

2 - Bass M. H., Denzer B. S. e Herman H. - Urobilin excretion in the anemias of infants and children. Am. Journ. Dis. Child. 27, 433, 1924.

3 - Czerny G. e Keller - "Des Kindes Ernährung, Ernährungstorunge. und Ernährungstherapie" - Leipzig e Wien, 1928.

4 - Glanzmann E. - Ernährung und Blutbildung. Schweiz. Med. Woch, $59,39,1929$.

5 - GyörgY P. - Beitrag zur Pathogenesis der Ziegenmilchanämie. Ztschr. f. Kinderh. 56, 1, 1934.

6 - Kohler G. O., Elvehjem C. A. e Hart E. B. - Goat's milk anemia. Am. Journ. Physiol. 113, 279, 1935.

7 - Letrerer E. - Das pathologisch-anatomische Bild des Ziegenmilch-anämie. Jahr. f. Kinderh. 130, 1, 1930.

8 - Marburg C. M. e Wiles H. O. - Granulocitopoietic fraction of yellow bone marrow. Arch. Int. Med. 61, 408, 1938.

9 - Oprtz H. - "Doenças do sangue e dos órgãos hematopoieticos" in Pfaundler e Schlossmann "Tratado de Pediatria” $2 .^{\circ}$ v. Trad. Bras. por Borges L. V., Edit. Guanabara, 1939.

10 - Mattar E., Soares de Almeida S., Mario Russo E., Jannini P., Сhapchap A. - Estudos de hematologia infantil (premio Alvarenga 1941) - traballio inédito.

11 - Rouminger E., Mayer H. e Bomskov C. - Anämiestudien am Wachsenden Organismus; uber die Pathogenese der Ziegenmilchanämie. Ztschr. f. d. Ges. Exp. Med. 33, 786, 1933.

12 - Sнонl A. T. - in "Textbook of Biochemistry" de Harrow e Sherwin Saunders, Filadelfia, 1935.

13 - SPÖRE H. V. - Berwerkungen uber Mangelanämien im Kindesalter mit besonderer Berückstigung der achylischen Chloroanämie. Arch. f. Kinderh. 111, 43, 1937. 


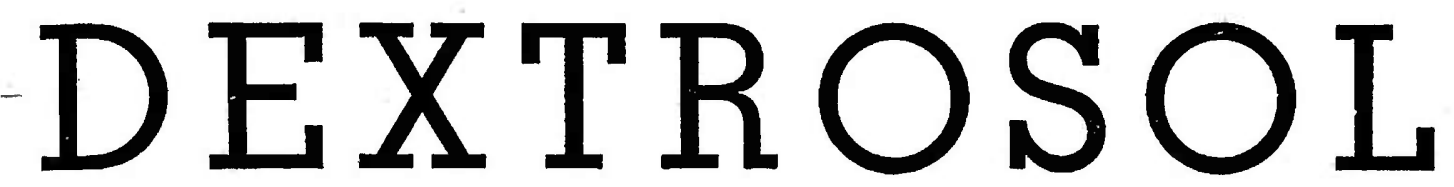

( $G L U C O S E-d)$

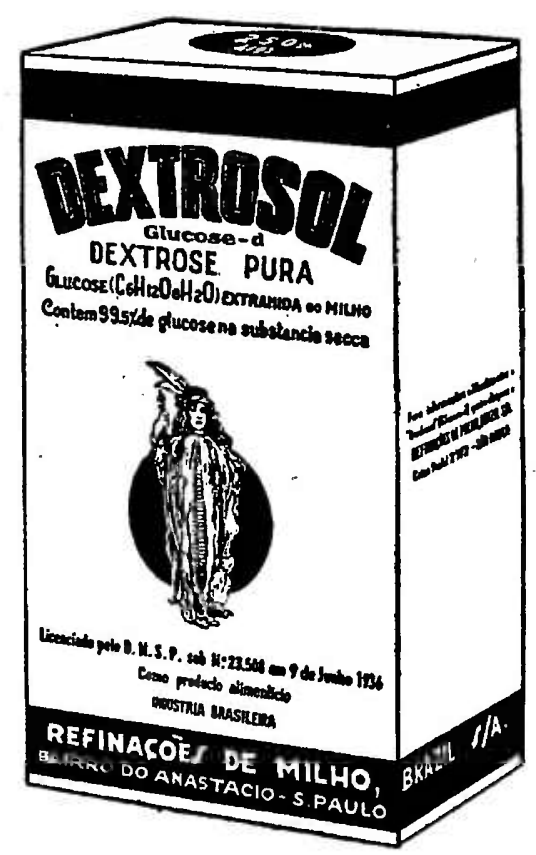

"DRENA AGUA dOS TECIDOS PARA A CIRCULAÇÃO, ELIMINANDO EDEMAS, AUMENTANDO O VOLUME SANGUINEO E PROMOVENDO A DIURESE"

E. MEYER - Usos Terapeuticos das Injeções Endovenosas de Soluções de Glucose) Zentralb. f. klin., Med. 102.343, 1925. Abst. J. A. M. A. 86.52I, 1926. 


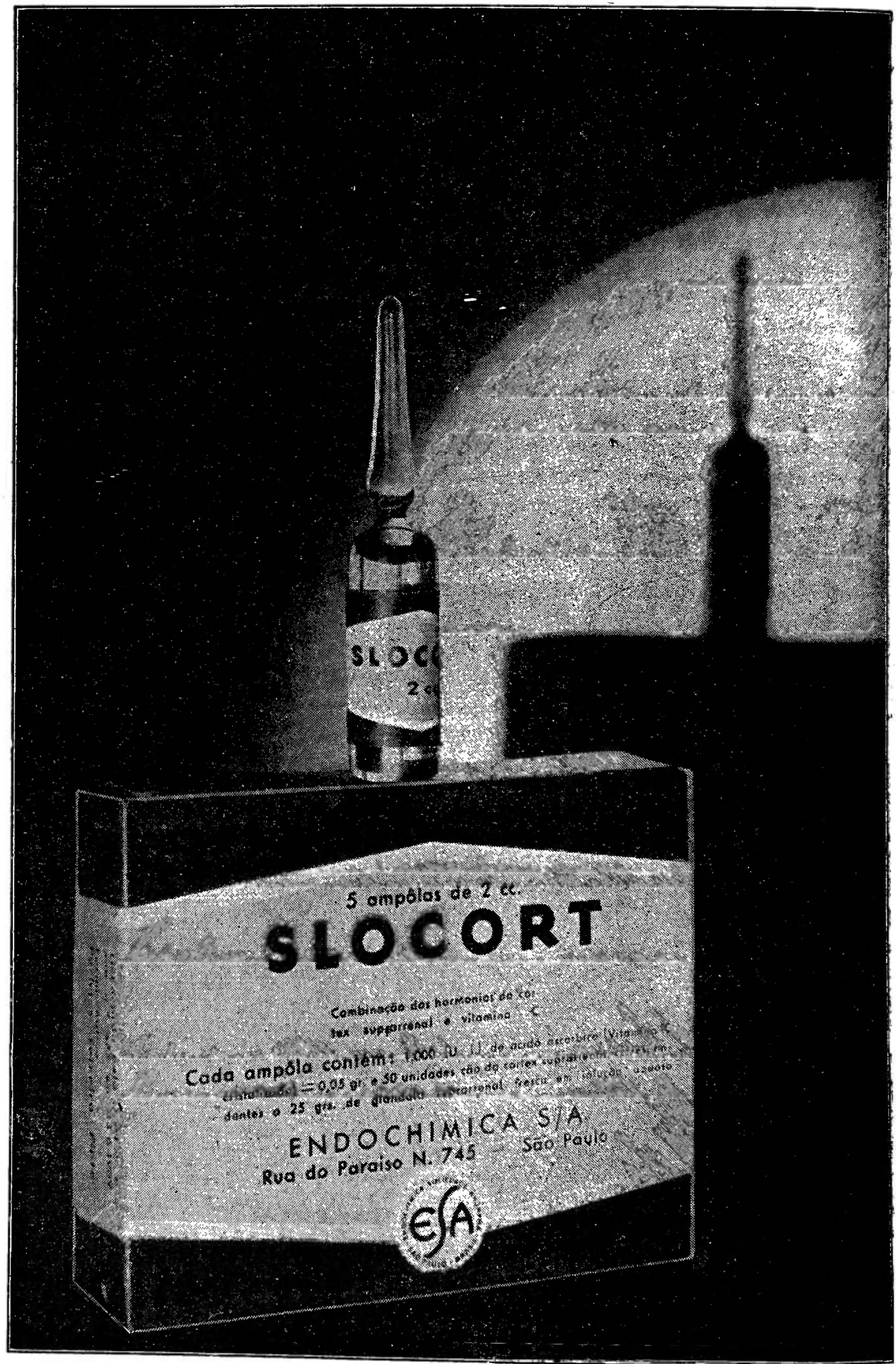

